\title{
Rheumatic Diseases and Treatment
}

\section{Foot Pain and Lesions in Systemic Sclerosis: Prevalence and Association with Organ Involvement}

\author{
Hadi Poormoghim ${ }^{1}$, Elham Andalib ${ }^{1}$, Arash Jalali ${ }^{2}$, Maryam Salimi-beni ${ }^{1}$ and Gholam Hossein \\ Ghafarpour ${ }^{1}$ \\ ${ }^{1}$ Iran University of Medical Sciences, Firoozgar Hospital, Iran \\ ${ }^{2}$ Department of Epidemiology and Biostatistics, Tehran University of Medical Sciences, Iran

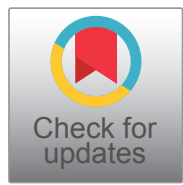

*Corresponding author: Hadi Poormoghim, MD, Iran University of Medical Sciences. Firoozgar Hospital, Tehran, Iran, Post code: 1593748711, Tel: +98-912-6439551

\begin{abstract}
Objective: Our goal was to evaluate prevalence of foot pain and lesions in patients with systemic sclerosis (SSc) and their association with other organ involvements.

Materials and methods: In this cross-section study 133 scleroderma patients were probed throughout a survey in which both forms of digital and non-digital plantar lesions were included. Chi-square test and student's t-test were used to determine the associations of foot pain and lesion with clinical features and serologic findings of the disease. multivariate analysis was used for determining independent factors associated with foot lesion and pain.
\end{abstract}

Results: Of all patients, 119 (89\%) were women with a mean age +Standard Deviation (SD) of $39.3+13.1$ years, $32(24.1 \%)$ patients had foot pain, and $40.6 \%$ were classified as having diffuse cutaneous SSc. Mean disease duration was $6.7 \pm 5.8$ years. Foot lesions were found in 47 (35\%) of patients; from which $30(93.8 \%)$ patients reported foot pain. In univariate analysis, Foot lesion were associated with vascular lesion, such as Raynaud 's phenomenon on the foot $(p<0.001)$, digital ulcer/gangrene $(p<0.005)$, calcinosis $(p<0.001)$, and high pulmonary arterial pressure on echocardiography (PAP), $(p<0.05)$. Additionally, we noticed the association of foot lesion with inflammatory disease, such as arthritis $(p<0.001)$, tendon friction rub ( $p$ $<0.004)$, pericardial effusion $(p<0.003)$, and esophageal dysmotility $(p<0.03)$ for vascular foot lesion. In the multivariate model, the diffuse subtype of the disease, presence of telangiectasia, calcinosis and Raynaud's on foot showed a significant association with vascular foot lesion.

Conclusion: Foot pain and lesion are common in Scleroderma patients, the diffuse subtype of the diseases, foot's Raynaud's, calcinosis, and telangiectasia were independently associated factors with foot lesion.

\section{Keywords}

Foot lesion, Foot pain, Systemic sclerosis

\section{Introduction}

Systemic Sclerosis (SSc) is a chronic devastating multi organ disease characterized by vascular abnormalities, fibrosis and immune dysregulation. Complications associated with upper extremities in SSc are well described, however, the foot problems are often neglected in scleroderma research. Foot ulcer has been noticed in rheumatoid arthritis, and diabetes, two diseases that pathogenesis of foot lesion seems close to SSc [1,2]. Even though, it has not been specifically studied, foot lesions in patients with scleroderma may have underlying pathophysiologic mechanisms like rheumatoid arthritis and diabetes [3-5].

It has been suggested that in rheumatoid arthritis mechanisms which could result in foot lesions include altered pressure distribution due to synovitis and deformity, fibro-fatty padding displacement on MTP, and plantar fat atrophy [6].

In patients with diabetes, foot lesions are the result of multiple factors and linked to a variety of risk factors such as peripheral neuropathy, vascular insufficiency and physiological measures [7]. A limited number of researches who previously studied lower extremities ulcer, did not specifically focused on foot pain or lesion 
$[3,8]$. Sari-Kouzel, et al. were among a few researchers, who reported nature of foot problems [4] in their SSC patients. They observed foot pain in $82 \%$, Raynaud's in $86 \%$ and digital ulcer in $26.1 \%$ of their subjects [4]. In SSc vascular complications such as Raynaud's phenomenon lead to digital ulcer, calcinosis (ulcer), gangrene, and amputation on foot. Mechanical complications such as hyperkeratotic lesion (corn and callous), could also result in foot lesions in SSc [9].

The goal of the current study was to carefully assess the prevalence and nature of foot complications in scleroderma patients who were registered in the study and further evaluate the association of foot problems with other organ involvements.

\section{Materials and Methods}

In this prospective cohort scleroderma, 133 patients, who were visited between Septembers and November 2014, were evaluated for presence of foot problems. Demographic, baseline, and paraclinical data were extracted from the institution's scleroderma registry. All patients had to meet the American College of Rheumatology/European League Against Rheumatism (ACR/EULAR 2013) classification criteria for SSc in order to be eligible for enrolment in the study [10]. The classifying subsets was done according to Le Roy, et al.'s study [11].

\section{Definition of organ involvement}

Vascular involvement was defined as presence of Raynaud's phenomenon observed by a physician, digital pitting ulcer, telangiectasia, ulceration or gangrene. Skin involvement detected on physical examination was scored based on the modified Rodnan Skin Score (mRSS). Musculoskeletal system involvement was defined as the presence of arthritis in more than one joint and palpable tendon friction rubs. Myositis was diagnosed when proximal muscle weakness was presented on physical examination or when any of the followings were present: Muscle biopsy confirming existence of myositis and electromyogram with a myopathic pattern or elevated serum enzymes reflecting a muscle disease. Gastrointestinal system involvement was defined as; esophageal dysmotility when esophageal dilatation was observed on the radiographic evaluation or based on manometry results. Pulmonary involvement included Interstitial Lung Disease (ILD) defined as presence of bilateral basilar fibrosis on chest radiography or high resolution computerized tomography (HRCT) scans and/or restrictive pattern on pulmonary function test that is, forced vital capacity (FVC) of less than $70 \%$ of predicted value, and PAP elevation measured by echocardiography $>40 \mathrm{mmHg}$. Cardiac involvement was defined as pericarditis and left ejection fraction of $<50 \%$ and/or arrhythmia requiring treatment. Renal involvement was defined when renal scleroderma malignant hypertension and/or rapidly progressive renal insufficiency and/ or microangiopathic hemolytic anemia were observed.

\section{Definition of foot problems}

All patients were questioned about foot pain or ulcer. They were asked to fill out a predesigned form by a rheumatologist (H.P) in order to document information on their foot problems. Patients, whose foot pain was reproducible with pressure on the lesion considered in the study, and those with foot pain related to plantar fasciitis or arthritis were excluded.

In addition to examination by a rheumatologist, a picture, which was taken from each foot lesion was reviewed by a dermatologist (GH. G). Both digital and non-digital plantar lesions were considered as foot lesions. Foot lesions were evaluated systematically in three areas of 1- forefoot (area that contains the five toes and metatarsal), 2- midfoot, and 3- hind foot (heel).

We classified foot lesions into two vascular and non-vascular (mechanical) lesions. Non-vascular lesions included hyperkeratotic lesions (corn, callous) that arises from mechanical pressure or friction on the skin $[12,13]$. A corn is a well-defined hyperkeratotic lesion with a central conical core of keratin that causes pain and inflammation. A callus is a diffuse hyperkeratotic area, with relatively even thickness and ill-defined margin. It is usually found under the metatarsal heads, at a site of friction, irritation, and pressure. Like hands, foot vascular lesions include toe tip pitting scar, telangiectasia, ulcer and gangrene or amputation and calcinosis. Mechanical foot lesions are hyperkeratotic lesions, callouses and corn formation in metatarsal, mid foot and

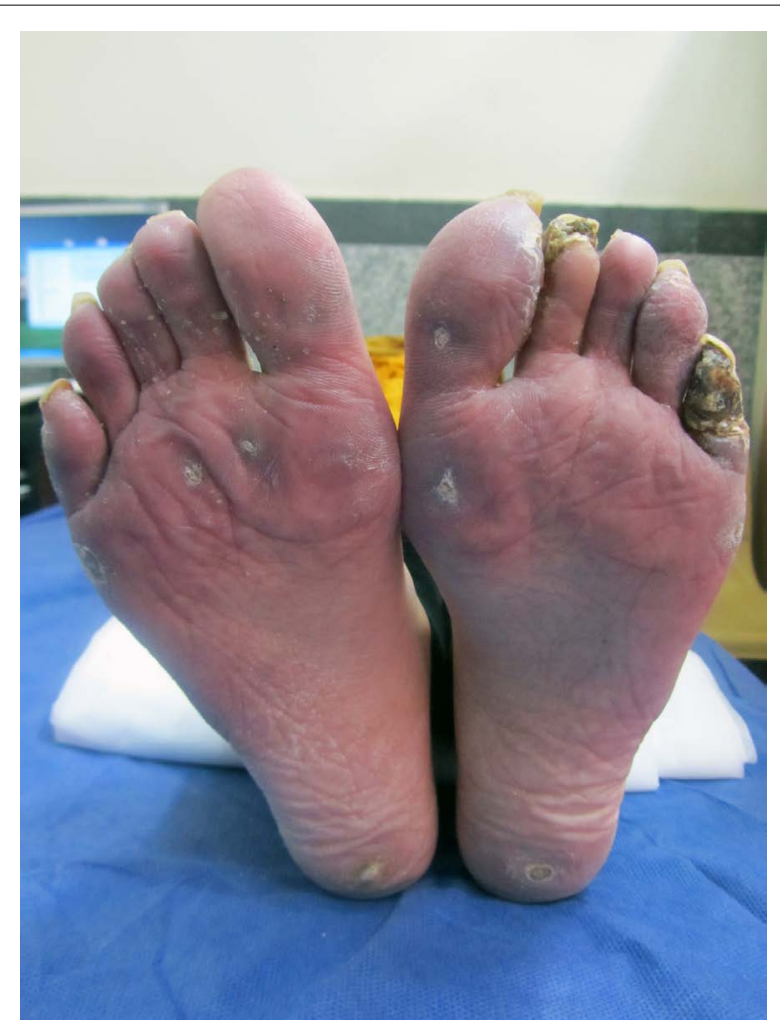

Figure 1: Figure shows foot lesions vascular and nonvascular. Gangrene on $5^{\text {th }}$ toe of left foot, hyperkeratotic lesion (corn, callous) on metatarsal area and heels. 
hind foot area. Figure 1 demonstrates digital lesions and non-digital plantar lesions.

\section{Serology studies}

Table 1: Demographic and Baseline data in 133 Systemic sclerosis patients with foot pain.

\begin{tabular}{|c|c|c|c|c|}
\hline Numbers of patients & $\begin{array}{l}\text { All } \\
133\end{array}$ & $\begin{array}{l}\text { Foot pain } \\
32(24.1)\end{array}$ & $\begin{array}{l}\text { No foot pains } \\
101(75.9)\end{array}$ & P value \\
\hline Age at time of diagnosis *(mean \pm SD/years) & $39.3 \pm 13.1$ & $34.5 \pm 11.3$ & $40.8 \pm 13.3$ & 0.01 \\
\hline Time interval from diagnosis to study *(mean \pm SD/years) & $6.7 \pm 5.8$ & $8.2 \pm 5.2$ & $6.3 \pm 5.9$ & 0.10 \\
\hline Gender: Female †No (\%) & $119(89)$ & $30(93.8)$ & $89(88.1)$ & 0.50 \\
\hline Diffuse subset No (\%) & $54(40.6)$ & $19(59.4)$ & $35(34.7)$ & 0.02 \\
\hline Raynaud's on foot (seen by physician) & $20(15.3)$ & $19(59.4)$ & $1(0.9)$ & 0.001 \\
\hline Foot lesion "No (\%) & $47(35.0)$ & $32(100.0)$ & $15(14.9)$ & 0.001 \\
\hline Foot vascular lesion & $31(23.3)$ & $23(71.9)$ & $8(7.9)$ & 0.001 \\
\hline Foot mechanical lesion & $40(30.1)$ & $30(93.8)$ & $10(9.9)$ & 0.001 \\
\hline
\end{tabular}

${ }^{*}$ mean \pm SD; means \pm Standard deviation; ${ }^{\dagger}$ No $(\%)$ : number (percentage); ${ }^{\top}$ No: number of patients with lesion.

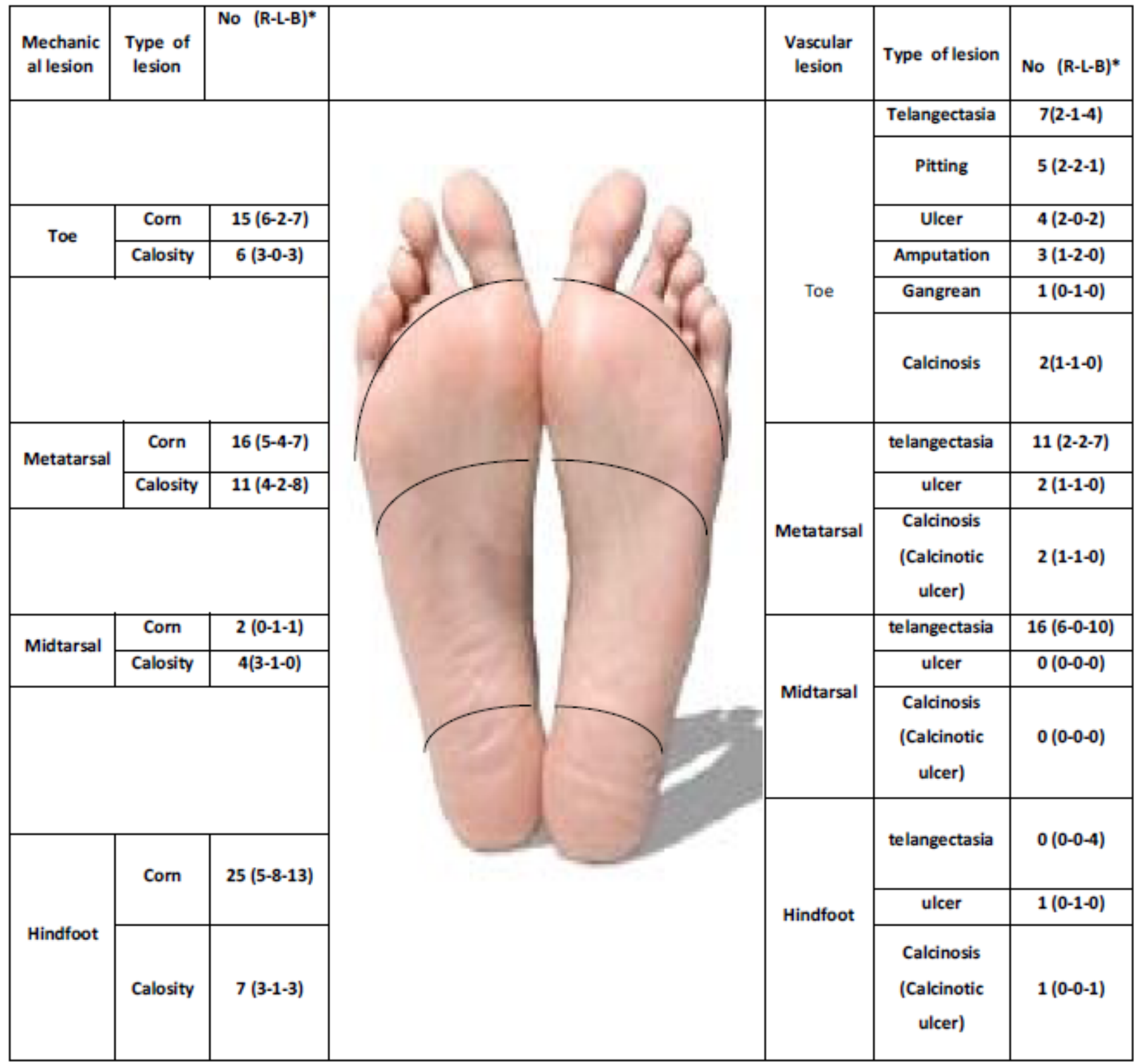

Figure 2: Classification and features of Foot involvement in 47 SSc patients with Foot lesion. 
detected by line immunoassay [Euroline systemic sclerosis profile (IgG), Euroimmune, Lubeck, Germany].

\section{Statistical analysis}

Chi-square and tails fisher exact test were used to compare categorical data. To evaluate the strength of association of data, Odds ratio (OR) with $95 \%$ confidence interval $(\mathrm{Cl})$ were used.

Student $t$-test was used to compare continuous variables and in the absence of normality assumption, the Mann-Whitney test analysis was performed. We used multivariate analysis for variables that showed a significant difference at level of $p<0.05$. A logistic regression analysis using forward method was performed to assess independent clinical or para-clinical factors associated with foot lesions. The Human Research Ethics Committee in our institution approved the study. Written informed consent was obtained from all participants.

\section{Results}

\section{Demographic features}

Out of 133 patients 32 (24\%) had foot pain which was severe enough to seek medical attention (Table 1 ). Patients with foot pain were younger than those without foot pain ( $34.5 \pm 11.3$ and $40.8 \pm 13.3$ respectively, $p<$ 0.01 ). Consistent with the scleroderma subjects (119) $89 \%$ of patients were female. We found no gender differences between the two groups with and without foot pain $(p<0.50)$. Diffuse cutaneous SSc subtype was more prevalent among patients with foot pain 19 (59.4\%) compared to patients without foot pain 35 $(34.7 \%)$, the difference was statistically significant $(p<$ $0.02)$. The mean disease interval time from diagnosis to the study, in patients with foot pain was $8.2 \pm 5.2$ (SD) years and in those without foot pain was $6.2 \pm 5.9$, ( $p<$ $0.10)$.

Prevalence of Raynaud's on foot in patients with foot pain compared to those without pain showed a significant difference, $(59.4 \%$ and $1 \%$ respectively, $p<$ 0.001). Overall, foot lesions were found in $47(35.0 \%)$ patients; from these cases, foot lesions in $32(100 \%)$ patients was accompanied with foot pain and in 15 (14.9\%) patients it was not, the mean difference was significant, $\mathrm{p}<0.001$.

Vascular lesions were found in 31 (23.3\%) patients in total, from those $23(71.9 \%)$ reported pain and $8(7.9 \%)$ had no foot pain. The difference was statistically significant $(p<0.001)$. Mechanical foot lesion was present in 40 (30.1\%) patients in total; 30 (93.8\%) of whom had foot pain and $10(9.9 \%)$ had no pain, $p<0.001$. Three patients who unfortunately, had amputation and three out of four patients with foot calcinosis reported foot pain in their examination.

\section{Classification and features of foot lesion}

Vascular foot lesions were observed proximally in toes and forefoot area. Mechanical foot lesions were prevalent in the metatarsal and hind foot. Figure 2 shows more details on patients' foot lesions.

Table 2: Correlation of vascular and mechanical Foot Lesion with organ system and Serological findings in 133 Patients with systemic sclerosis (SSc).

\begin{tabular}{|c|c|c|c|c|c|c|}
\hline & $\begin{array}{l}\text { Missing } \\
\text { value }\end{array}$ & $\begin{array}{l}\text { No \% } \\
\text { frequency of } \\
\text { Factors }\end{array}$ & $\begin{array}{l}\text { Associated with } \\
\text { Foot vascular } \\
\text { lesion } \\
\text { OR }(\mathrm{Cl} 95 \%)\end{array}$ & $\mathrm{P}$ value & $\begin{array}{l}\text { Associated with } \\
\text { Foot mechanical } \\
\text { lesion OR (Cl 95\%) }\end{array}$ & $P$ value \\
\hline $\begin{array}{l}\text { age } \\
\text { Female: Male } \\
\text { Subtypes of disease } \\
\text { Time interval from SSc diagnosis } \\
\text { to study } \\
\text { Foot pain }\end{array}$ & $\begin{array}{l}0 \\
0 \\
0 \\
0 \\
0\end{array}$ & $\begin{array}{l}133(100) \\
133 \\
133 \\
133 \\
32\end{array}$ & $\begin{array}{l}0.97(0.94-1.01) \\
0.88(0.23-3.40) \\
2.53(1.11-5.76) \\
1.04(0.98-1.11) \\
29.7(10.3-85.4)\end{array}$ & $\begin{array}{l}0.18 \\
0.86 \\
0.02 \\
0.17 \\
0.001\end{array}$ & $\begin{array}{l}0.98(0.95-1.01) \\
0.92(0.27-3.13) \\
2.01(0.94-4.26) \\
1.03(0.97-1.10) \\
136.5(28.3-658.2)\end{array}$ & $\begin{array}{l}0.23 \\
0.89 \\
0.07 \\
0.24 \\
0.001\end{array}$ \\
\hline $\begin{array}{l}\text { Raynaud's on hands seen by } \\
\text { physician } \\
\text { Telangectasia face/mouth/hand } \\
\text { Hand Digital tip scar } \\
\text { Hand Digital ulcer/gangren } \\
\text { Digital (upper and lower) } \\
\text { amputation } \\
\text { Calcinosis in any site } \\
\text { Raynauds on foot (seen by } \\
\text { physician) }\end{array}$ & $\begin{array}{l}1 \\
0 \\
0 \\
0 \\
0 \\
0\end{array}$ & $\begin{array}{l}71(53.8) \\
92(69.2) \\
73(54.9) \\
42(31.6) \\
14(10.9) \\
25(18.8) \\
20(15.3)\end{array}$ & $\begin{array}{l}0.79(0.35-1.79) \\
2.84(1.00-8.02) \\
2.99(1.22-7.30) \\
2.91(1.27-6.65) \\
2.81(0.89-8.86) \\
5.41(2.19-13.7) \\
17.8(5.68-55.8)\end{array}$ & $\begin{array}{l}0.58 \\
0.04 \\
0.01 \\
0.009 \\
0.07 \\
0.001 \\
0.001\end{array}$ & $\begin{array}{l}0.63(0.29-1.36) \\
1.25(0.55-2.84) \\
2.48(1.13-5.47) \\
4.65(2.07-10.0) \\
5.27(1.64-17.0) \\
4.01(1.62-9.91) \\
36.4(7.85-168.0)\end{array}$ & $\begin{array}{l}0.24 \\
0.58 \\
0.02 \\
0.001 \\
0.003 \\
0.002 \\
0.001\end{array}$ \\
\hline $\begin{array}{l}\text { Hx of esophageal reflux } \\
\text { Esophageal dysmotility } \\
\text { Diarrhea/malabsorption }\end{array}$ & $\begin{array}{l}0 \\
8 \\
1\end{array}$ & $\begin{array}{l}110(82.7) \\
60(48.0) \\
28(21.0)\end{array}$ & $\begin{array}{l}0.83(0.29-2.33) \\
1.58(0.69-3.61) \\
1.11(0.42-2.92)\end{array}$ & $\begin{array}{l}0.72 \\
0.27 \\
0.83\end{array}$ & $\begin{array}{l}1.68(0.57-4.89) \\
2.42(1.10-5.32) \\
1.37(0.56-3.31)\end{array}$ & $\begin{array}{l}0.33 \\
0.02 \\
0.48\end{array}$ \\
\hline
\end{tabular}




\begin{tabular}{|l|l|l|l|l|l|l|}
\hline Arthritis & 0 & $18(13.5)$ & $5.59(1.97-15.8)$ & 0.001 & $8.47(2.77-25.9)$ & 0.001 \\
\hline Myositis & 3 & $42(32.3)$ & $1.28(0.54-3.03)$ & 0.56 & $1.64(0.75-3.57)$ & 0.20 \\
\hline Tendon friction rub & 0 & $29(21.2)$ & $3.15(1.29-7.69)$ & 0.01 & $4.10(1.73-9.71)$ & 0.001 \\
\hline Lung fibrosis (ILD) & 2 & $89(67.9)$ & $1.74(0.68-4.46)$ & 0.24 & $2.16(0.89-5.24)$ & 0.84 \\
\hline Advanced pulmonary fibrosis & 2 & $34(25.7)$ & $1.30(0.52-3.21)$ & 0.56 & $1.02(0.43-2.42)$ & 0.95 \\
\hline PAP $\geq \mathbf{4 0}$ on echocardiography & 6 & $15(11.8)$ & $3.58(1.17-10.9)$ & 0.03 & $3.27(1.09-9.81)$ & 0.04 \\
\hline Pericardial effusion & 6 & $13(10.2)$ & $5.16(1.57-16.9)$ & 0.004 & $2.51(0.78-8.08)$ & 0.11 \\
\hline Heart failure/cardiomyopathy & 10 & $10(8.1)$ & $2.60(0.67-10.0)$ & 0.15 & $1.13(0.27-4.66)$ & 0.83 \\
\hline Renal crisis & 0 & $3(2.3)$ & $1.66(0.14-19.0)$ & 0.67 & $1.16(0.13-13.2)$ & 0.90 \\
\hline HCT $<30 \%$ & 0 & $15(12.4)$ & $0.84(0.24-2.90)$ & 0.79 & $0.56(0.18-1.72)$ & 0.32 \\
\hline ESR $>\mathbf{3 0}$ & 0 & $57(42.9)$ & $2.23(1.00-5.06)$ & 0.05 & $0.97(0.46-2.72)$ & 0.95 \\
\hline ANA & 12 & $106(87.6)$ & $0.84(0.24-2.90)$ & 0.80 & $0.56(0.18-1.72)$ & 0.30 \\
\hline ACA & 36 & $12(12.4)$ & $1.08(0.26-4.39)$ & 1.00 & $0.67(0.17-2.70)$ & 0.58 \\
\hline Anti-Topo & 34 & $71(71.7)$ & $0.94(0.34-2.60)$ & 1.00 & $1.53(0.57-4.10)$ & 0.40 \\
\hline
\end{tabular}

Table 3: Independent factors associated with vascular and mechanical foot lesion in multivariate analysis.

\begin{tabular}{|l|l|l|l|}
\hline Vascular lesion & B & OR (95\% Cl) & P value \\
\hline Subtype (diffuse) & 1.385 & $3.99(1.12-14.2)$ & 0.03 \\
\hline Telangectasia & 1.43 & $4.17(1.01-17.4)$ & 0.05 \\
\hline Calcinosis & 2.12 & $8.349(1.95-35.6)$ & 0.004 \\
\hline Raynauds' on foot & 2.53 & $12.6(2.92-54.4)$ & 0.001 \\
\hline Mechanical lesion & & & 0.04 \\
\hline Esophageal dysmotility & 1.06 & $2.90(1.06-7.93)$ & 0.03 \\
\hline Digital ulcer on hand & 1.08 & $2.94(1.10-7.88)$ & 0.001 \\
\hline Raynauds' on foot & 2.97 & $19.65(3.74-103.26)$ & \\
\hline
\end{tabular}

We found no differences according to age, gender, disease subsets, objective Raynaud's phenomenon, myositis, $\mathrm{Gl}$ symptoms, renal function tests, $\mathrm{EF}<50$ measures, lung fibrosis and autoantibodies in the two groups of patients. Association of clinical feature and serological findings in univariate analysis is shown in Table 2.

In the multivariate model, the diffuse subtype of disease, presence of telangiectasia, calcinosis and Raynaud's on foot kept a significant association with vascular foot lesion. Presence of esophageal dysmotility, amputation and foot Raynaud's were independent factors associated with mechanical foot lesion (Table 3).

\section{Discussion}

The current cross-sectional study demonstrated that foot pain is a prevalent clinical symptom of SSc. Clinicians should not only look for it but also take it as a very serious sign in disease management. Foot lesions which were seen in one third of patients were mostly accompanied with pain. The results of this study highlight the importance of foot pain and lesion as contributing factors to morbidity in patients with SSc and must carefully be assessed. This study showed the correlation of vascular and mechanical foot lesions with organ involvement was closely similar. We noticed that some patients, who had foot lesions, did not have any complain about foot pain; this may be due to mid-foot lesion with less pressure effect.
In the current study, foot lesions mostly showed an association with vascular and inflammatory manifestation of disease. Vascular manifestation of disease on hands as well as Raynaud's phenomenon on foot and PAP $>40 \mathrm{mmHg}$ were more prevalent in patients with foot complications. this study did not find any association between foot lesion and Scleroderma Renal Crisis (SRC). This may be due to limited number of patients with SRC in the study. To our knowledge, there is no study that has evaluated the association of organ involvement with foot lesion in SSc.

We found correlation of esophageal dysmotility symptoms with mechanical foot lesion. Although causality of esophageal dysmotility is not well known, vascular damage, neuromuscular dysfunction and fibrosis are considered as pathogenic causes of esophageal dysmotility $[14,15]$.

In a univariate analysis, correlation of inflammatory presentation of disease such as arthritis, palpable tendon friction rubs, pericarditis and elevated ESR with foot lesions was found. The inflammatory nature of arthritis in scleroderma patients was supported by previous studies and through results from synovial biopsy [16] or other new imaging tools [17]. It was shown that tenosynovitis in SSc can be caused by inflammatory or fibrotic changes in tendon sheath [17]. In addition, in histological investigation, chronic inflammation was reported as the leading cause of pericardial disease in SSc patients [18]. 
High prevalence of anti-centromere Abs was shown to be a predictive factor for digital ischemic event and digital loss in scleroderma patients [19]; however, in this study we were not able to find such relationship. This could be due to low prevalence of ACA in current study.

We found an association between foot lesion and diffuse subset of disease in univariate analysis and multivariate analysis. On the other hand, diffuse subset was also the independent factor associated with vascular foot lesion. It could be speculated that patients with diffuse disease had more severe vascular presentation of disease. Vascular features of disease, such as calcinosis, Raynaud's on foot, and telangiectasia also showed an independent association with foot vascular lesion. Interestingly, we found association between mechanical foot pain and some vascular features of the disease. Based on these findings and extrapolating from ischemia as cause of digital fat pad atrophy on hands' fingers, the authors speculated that the same mechanism may result in foot fat pad atrophy.

There were limitations in this study. We calculated point prevalence of foot lesion and foot pain and did not conduct a numerical scoring. The cumulative incidence of foot lesion may be higher than reported in the study. Another limitation was that macrovascular disease in the lower extremities was not studied. Moreover, in the patients with high PAP in echocardiography, catheterization was not conducted as part of diagnosis of pulmonary hypertension.

Foot problems are very prevalent and should be considered as part of routine clinical management of SSc. The current study, for the first time, demonstrated an association between foot pain and foot lesions as well as foot lesion with clinical and para-clinical features of SSc. In evaluation of patients with foot pain and lesion, patients with diffuse subset of disease and vascular manifestation on hands, Raynaud's phenomenon on foot, and inflammatory disease presentation should be evaluated carefully for foot pain and lesion.

\section{Conflict of Interest}

None.

\section{References}

1. van der Leeden M, Steultjens M, Dekker JH, Prins AP, Dekker $\mathrm{J}$, et al. (2006) Forefoot joint damage, pain and disability in rheumatoid arthritis patients with foot complaints: the role of plantar pressure and gait characteristics. Rheumatology 45: 465-469.

2. Dalal S, Widgerow AD, Evans GR (2013) The plantar fat pad and the diabetic foot - a review. Int Wound J 12: 17421801.

3. Shanmugam VK, Price P, Attinger CE, Steen VD (2010) Lower Extremity Ulcers in Systemic Sclerosis: Features and Response to Therapy. Int J Rheumatol.

4. Sari-Kouzel H, Hutchinson CE, Middleton A, Webb F, Moore
T, et al. (2001). Foot problems in patients with systemic sclerosis. Rheumatology 40: 410-413.

5. Reiber GE, Vileikyte L, Boyko EJ, del Aguila M, Smith DG, et al. (1995) Causal pathway for in incident lower extremity ulcers in patients with foot ulcer from two setting. Diabetes care 1: 157-162.

6. Alcacer-Pitarch B, Buch MH, Gray J, Denton CP, Herrick A, et al. (2012) Pressure and pain in scleroderma an evaluation of simple intervention (PISCES): randomised controlled trial protocol. BMC Musculoskelet Disord 13: 11.

7. Crawford F, Inkster M, Kleijnen J, Fahey T (2007) Predicting foot ulcers in patients with diabetes: A systematic review and meta-analysis. QJM 100: 65-86.

8. Reidy ME, Steen V, Nicholas J (1992) Lower extremity amputation in scleroderma. Arch Phys Med Rehabil 73: 811813.

9. Chathra N, Bhat RM (2017) Corn in scleroderma. Indian Dermatol Online J 8: 49-50.

10. van den Hoogen F, Khanna D, Fransen J, Johnson SR, Baron M, et al. (2013) 2013 classification criteria for systemic sclerosis: an American College of Rheumatology/European League against Rheumatism collaborative initiative. Ann Rheum Dis 65: 2737-2747.

11. LeRoy EC, Medsger TA Jr. (2001) Criteria for the classification of early systemic sclerosis. J Rheumatol 28: 15731576.

12. Singh D, Bentley G, Trevino SG (1996) Callosities, corns, and calluses Callosities, corns, and calluses. BMJ 312: 1403-1406.

13. Freeman DB (2002) Corns and Calluses Resulting from Mechanical Hyperkeratosis. Am Fam Physician 11: 22772280.

14. Korn JH, Mayes M, Matucci Cerinic M, Rainisio M, Pope J, et al. (2004) Digital ulcers in systemic sclerosis: prevention by treatment withbosentan, an oral endothelin receptor antagonist. Arthritis Rheum 50: 3985-3993.

15. Sjogren RW (1994) Gastrointestinal motility disorders in scleroderma. Arthritis Rheum 37: 1265-1282.

16. Schumacher HR Jr. (1973) Joint involvement in progressive systemic sclerosis (scleroderma): a light and electron microscopic study of synovial membrane and fluid. Am J Clin Pathol 60: 593-600.

17. Elhai M, Guerini H, Bazeli R, Avouac J, Freire V, et al. (2012) Ultrasonographic hand features in systemic sclerosis and correlates with clinical, biologic, and radiographic findings. Arthritis Care Res 8: 1244-1249.

18. Byers RJ, Marshall DA, A J Freemont AJ (1997) Pericardial involvement in systemic sclerosis. Ann Rheum Dis 56: $393-$ 394.

19. Herrick AL, Heaney M, Hollis S, Jayson MI (1994) Anticardiolipin, anticentromere and anti-Scl-70 antibodies in patients with systemic sclerosis and severe digital ischemia. Ann Rheum Dis 53: 540-542. 